\title{
Preliminary software architecture for the PRIMA astrometric Data Analysis Facility
}

\author{
Jeroen A. de Jong ${ }^{a}$, Eric J. Bakker ${ }^{a}$, Walter Jaffe ${ }^{a}$ and Sabine Frink ${ }^{a}$ \\ ${ }^{a}$ Leiden Observatory, P.O. Box 9513, 2300 RA Leiden, The Netherlands
}

\begin{abstract}
One of the goals of the VLTI PRIMA (Phase Referenced Imaging and Micro-arcsecond Astrometry) facility will be to obtain high accuracy astrometry (of the order of $10 \mu \mathrm{arcsec}$ ) for the measurement of the reflex motion due to planets. In order to achieve this an offline astrometric Data Analysis Facility (DAF) is planned to perform a homogeneous and iterative analysis over several years of observations. This system will be part of the PRIMA Data Reduction Library (DRL), which also contains the online pipeline. The most important module of the DAF will be the Trend Analysis to identify and fit the systematic errors and feed them back into the data reduction. This requires an infrastructure which allows for comprehensive access to all raw and derived data and enough flexibility to easily introduce new algorithms in the system. We plan to realize this with a database, sophisticated middleware and Application Programmers' Interfaces (APIs) for the algorithms and user interface plug-ins. We present in this paper the requirements and preliminary design of the DAF, as well as the implementation issues concerning the integration with other modules of the DRL and ESO compliance.
\end{abstract}

Keywords: VLTI, PRIMA, astrometry, planet search, data analysis, software design

\section{INTRODUCTION}

In less than 10 years after the first detection of a planet orbiting another star, more than 100 giant extra-solar planets have been discovered. This avalanche of results has opened a very exciting field of research: exploration of the characteristics of other planetary systems. The discoveries of the past few years have stimulated new planetary formation models leading to a new picture of planet formation.

So far, all extra-solar planets found around stars in the vicinity of the Sun have been detected by radialvelocity measurements. Large surveys are conducted in both hemispheres with about $3000 \mathrm{G}, \mathrm{K}$ and early M stars being monitored regularly. While these surveys are very successful, their intrinsic biases imply some limitations both on the detection sensitivity and the data interpretation. In particular, this method is restricted to certain types of stars and leaves the inclination angle of the orbit $i$ undetermined, thus providing only a lower limit to the mass of the planets.

The development of state-of-the-art optical long-baseline interferometric instruments like the Very Large Telescope Interferometer on Cerro Paranal opens new perspectives in this research area. The European Southern Observatory (ESO) is currently acquiring the hardware for PRIMA, a facility that will enable astrometric observations with the Very Large Telescope Interferometer (VLTI). The PRIMA facility holds the promise to carry out an exciting program on the astrophysics of extra-solar planets through precise astrometry, which is a very complementary technique to the radial-velocity method. It has a different detection bias, favoring planets in large orbits versus the short-period orbits preferentially detected by the radial-velocity technique. Moreover, astrometry measures two components (right ascension and declination) of the stellar reflex motion versus the single radial component that is observable spectroscopically. However, to play a significant role, an astrometric accuracy of order $10 \mu \mathrm{arcsec}$ is needed, which is beyond the performance of current instrumentation (although the PTI reached a similar accuracy for the limited case of very close binaries $\left.(0.25 \text { " separation })^{1}\right)$. Besides the hardware, which is currently being developed, a sophisticated calibration plan and data analysis software will be required to reach this accuracy. Next to the search and characterization of extrasolar planets, other science drivers will be the motion of stars in clusters and the galactic center and graviational lensing. ${ }^{2}$

Send correspondence to jdejong@strw.leidenuniv.nl 
The Earth's atmosphere puts fundamental limitations on any astrometry from the ground and causes a typical image motion of about 0.5 ". However, over small angles in the sky the image motion is highly correlated and differential positional measurements of objects in the same field are much more accurate. For a 100m baseline and a separation of 10 arcsec one would expect to achieve on Paranal $10 \mu$ arcsec accuracy in a half-hour integration.

PRIMA is a dual-star interferometer for the VLTI. Each telescope sends two separate beams from a science and reference target into the delay lines. The delay difference between the two fields $\left(\Delta D_{\text {int }}\right)$ is taken out with an additional short-stroke differential delay line, and monitored with an internal laser metrology system. The total differential delay $\Delta D$ is obtained after addition of the delay difference between the two fringe sensor units. With $\Delta D$ and a good knowledge of the projected baseline one can derive the coordinate difference between the target and reference star. The difficult challenge will be to compute the baselines well enough from a large number of calibration measurements.

The PRIMA astrometry project is divided into the hardware Differential Delay Line (DDL) component and an Astrometric Operations and Software (AOS) component. The other components of PRIMA (Star Separator, Fring Sensor Units) will be provided by ESO and its subcontractors. The PRIMA AOS project will be carried out by a consortium which includes Leiden Observatory, Geneva Observatory and MPIA Heidelberg and provides the following categories of deliverables:

- PRIMA Astrometry Error Budget, Operations and Calibration Strategy

- PRIMA Astrometry Observation Preparation Tools

- PRIMA Astrometry Data Reduction Library

The Data Reduction Library (DRL) provides the software for the proper reduction and calibration of the PRIMA astrometric data. It consists basically of a pipeline reduction package for online and offline batch reduction and a Data Analysis Facility (DAF). The scope of this paper is limited to the DAF and its external interfaces to the rest of the DRL.

The output of the PRIMA astrometric data reduction will consist of the projected angular separations and accompanying computed baselines. In order to get accurate positions from these measurements a large number of reference sources with reasonably well known properties should be observed in a systematic way. This implies a number of constraints on the target selection and observation scheduling ${ }^{3}$ :

1. Science targets should be observed frequently at epochs which place the largest number of constraints on the astrometric solution.

2. A set (about 10, evenly spread over the accessible RA-DEC range) of visual binaries should be selected, with well known orbital parameters, to assess the accuracy of the astrometric parameters.

3. A set of baseline calibrators will be identified (up to 100, evenly spread over the accessible RA-DEC range) which will be monitored on a regular basis.

The angular separations must be solved as function of at least the parallax and proper motion. Furthermore, a large number of unknown and known systematic errors on the optical path difference need to be derived from all the available data. The fitted errors must be adjusted everytime new data arrives into the system and the parameter space may be subject to change when new trends in the data are discovered.

\section{REQUIREMENTS}

The next sections will describe the requirements which the scientific goals pose on the DAF. 


\subsection{Technical requirements}

The system must cope with several years of data and provide full access to all raw and intermediate data in order to give the astronomers the necessary insight for finding the systematic errors. This information base should be flexible enough to allow for the addition of new derived information when a new analysis method is added to the system. Considering the large number of computations involved in the analysis, the system should be as efficient as possible with recomputations. Therefore, it should keep track of which steps in the analysis have been altered and only recompute these steps and those depending on them. The database model must contain proper relations in order to achieve this. Processing should be possible interactively and non-interactively. The reduction time of a single exposure should be short enough to allow for a reasonable number of parameter modification tests.

The package should be self-contained and distribution of the package should be possible after a detailed development phase. Technical requirements from this are that the software package DAF should have an autoinstall procedure, it should run on a standard UNIX based computer, and should not rely on commercial tools (e.g. IDL). In order to guarantee robustness scripting languages should only be used for peripheral user interface related code.

\subsection{Calibration requirements}

Calibration of astrometric observables is a key task for the DAF. Many systematic errors are expected and the astrometric observables must be calibrated. Requirements on the calibration are that there should be tools available that allows to:

- Reduce the data of calibrators (wide binaries, and a set of baseline calibrators);

- To compute the expected astrometric observables for the calibrators;

- To correlate the observed versus computed error with a large number of critical (instrumental) parameters and determine the correlation coefficients;

- Visualization tools to interactively identify trends.

- Tools for integrating new error correction procedures into the DAF.

The DRL should allow calibration of any given exposure which is in the archive, without access to any of the other data. For this, a calibration engine will need to be developed. Requirements on the calibration engine are among others:

- It should contain all the necessary calibration parameters such that the ESO pipeline can calibrate any given exposure;

- It should be sufficiently flexible to allow regular updates of the calibration engine, without needing to update the ESO pipeline.

\subsection{User interface}

It is clear that the requirements on both flexibility and robustness pose a considerable challenge to the user interface design. The user interface needs to provide a uniform and comprehensive view on all the available data in graphical and tabular form. However, it should also provide the flexibility for adding easily new views and analysis steps on the data. These additions should always be integrated through uniform APIs (application programmers' interfaces) in order to maintain a consistent codebase. The number of APIs should be as small as possible and they should be available as abstract base classes. APIs will be provided for creating plugin panels to the user interface and adding an accompanying step to the reduction chain. The user interface plugins will include tabular and graphical visualizations and input forms, as well as interactive GUI components where needed. 


\subsection{Interfaces to data and algorithms}

The input data will be provided as FITS files of which the format has to be specified by the PRIMA consortium. The software must be able to handle all standard IAU FITS format data (including ESO extensions). The internal data of the DAF will be stored in a database and XML files in case of saved configurations and snapshots. Data products and calibration files will be exported as FITS files.

Algorithms will be kept strictly separate from the infrastructure in order to be able to share them between the DAF and the pipeline. All algorithms will be written in ANSI C.

\subsection{Quality control}

The DAF should verify the performance of each PRIMA subsystem as far as the data available in the FITS files allow. It should make an assessment of the performance of these subsystems, and possible systematic errors. For example, the performance of the Fringe Sensor Unit (FSU) should be assessed by taking into account the spectrum of the star, the correlation between the fringe tracking parameters for both FSUs, and whether the observed signal from the FSU is consistent with that expected for the program stars (e.g. the ABCD signal of the FSU should be compared with the predicted signal given the angular size of the program star).

\subsection{Simulations}

Each software module should have a simulation mode. A PRIMA astrometry data simulator should be available to allow to test the DAF before PRIMA astrometry data will be available. This simulator should be sophisticated enough to test the data flow and algorithms. Especially the trend analysis procedures (see Sect. 3.3) should be tested by providing data with combinations of multiple known systematic errors. This should provide limits on how well the algorithms can disentangle these errors.

\section{PRELIMINARY DESIGN}

To meet the requirements specified in the previous section we foresee the following modules in the DAF:

1. Database: Contains all information about the raw and intermediate data: FITS headers, dependencies between steps, results of computations and references to intermediate files.

2. Analysis Chain Manager (ACM): Sets up the data analysis chains for the input data according to the available recipes. Manages branches of different recipes for trying out algorithms.

3. Data Reduction Algorithms (DRA): The core algorithms for the astrometric data reduction.

4. Trend Analysis (TA): Set of tools and algorithms for identifying and fitting trends and systematic errors in the data.

5. Sequencer: Executes the DRA and TA and stores the results in the database.

6. Mathematical library: Provides all the routines for the numerical computations (fitting, FFT, optimization, interpolation, etc.)

7. Calibration Engine (CE): Generates the calibration matrix (CM) for external usage in the archive and online pipelines.

8. Server: Web application server which provides the user interface.

These modules provide a client-server approach in which the user interface runs within a web browser. This has the advantage that all data can be stored and processed on a single (cluster of) computer(s). No installations on the client side are necessary, except for some browser plug-ins. We consider this the most logical approach since the amount of data is too large (order of a terabyte) to distribute over different sites, while the operators must be able to access the DAF from anywhere.

The relations between these modules is shown in Fig. 1. Besides these modules there are several external interfaces to the ESO software: the DRA are also used in the ESO pipeline and the CM must be read by this pipeline. 


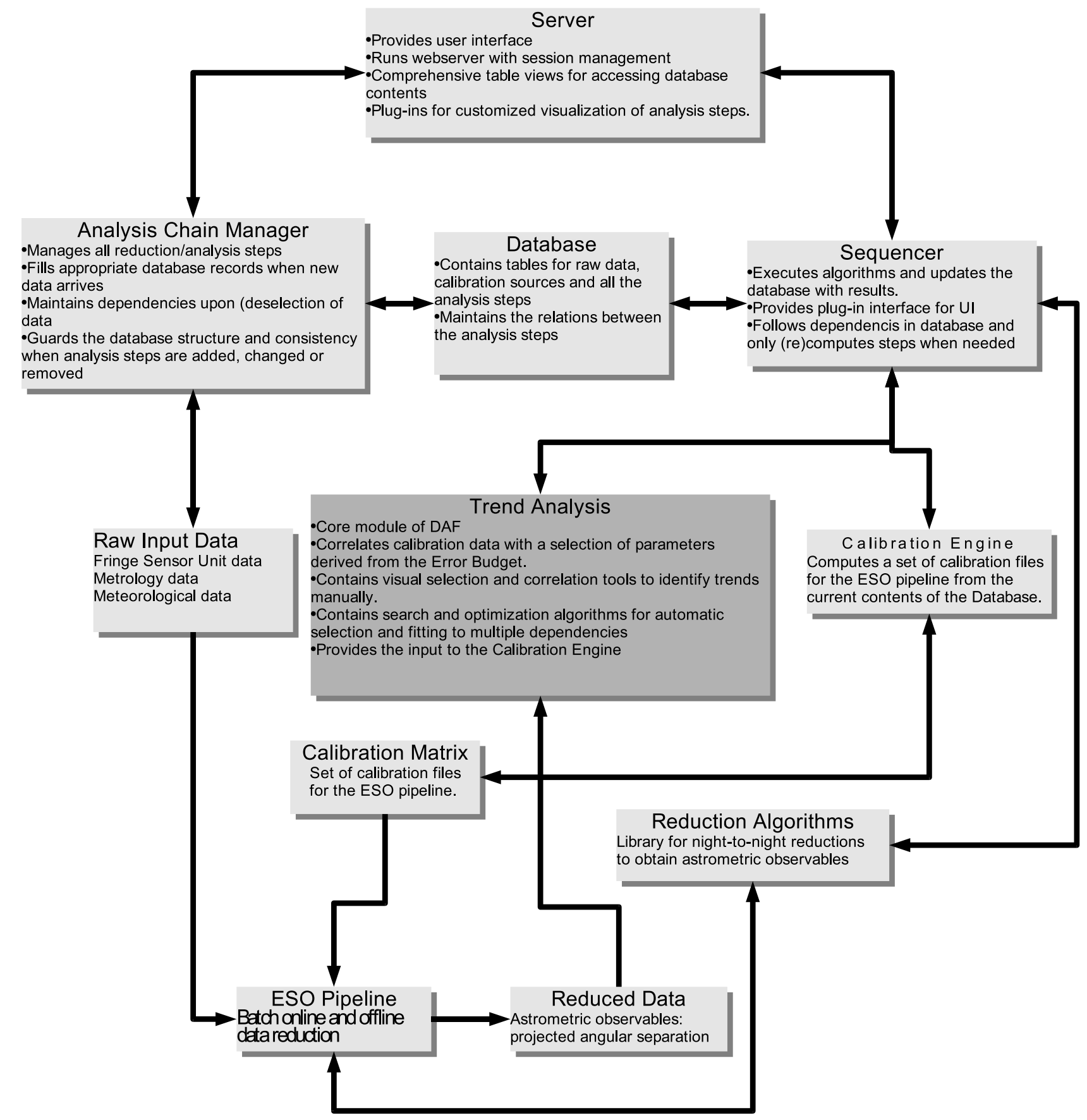

Figure 1. Overview of the DAF and its relations to other parts of the PRIMA DRL. 


\subsection{Database}

The database cannot have a static model, since changes in the analysis recipe have to be incorporated. These changes will inevitably lead to new tables and alterations in the relations between the tables. Therefore, we describe here only an abstract model. The actual model will be maintained by the ACM based on the configuration files which describe the current data analysis chains. The database will contain the following table groups:

Raw Data tables These tables contain information about the input data. This includes the organization in Observation Blocks and of each raw data file the date, time, type of observation and other relevant FITS keywords (to be specified in a configuration file).

Parameter tables Contain the actual parameter values used in the algorithms. All these parameters refer to the step they belong to.

Analysis step tables Each reduction or analysis step has its own table in which the dependencies on other steps and results are stored. These tables are coupled to algorithms from either the DRA or TA.

Source tables Contains the astrophysical information about the science and calibration sources (e.g. color, spectral type, orbital elements, proper motion, parallax)

Administration tables Contain configuration information as obtained from the configuration files. They are mainly required for integrity checks.

The SQL queries will be concentrated as much as possible in an object to relational mapping package with a uniform API. This allows for optimizing the queries without affecting higher level code.

\subsection{Data Reduction Algorithms}

This module contains all the algorithms for the pipeline reduction. The module will be used in the DAF to get intermediate reduction data in the database and to allow for the trend analysis module to redo reductions in order to improve the calibration. The module has to be fully compliant with the ESO pipeline standards (coded in ANSI-C and compatible with the Common Pipeline Library $(\mathrm{CPL})^{4}$ ). For each step an algorithm will be developed and an entry will be created in the reduction recipe configuration file. The DAF takes care of the database table and associations. Figure 2 shows a preliminary diagram of the reduction recipe.

\subsection{Trend Analysis}

This is the most important module of the DAF. It contains the algorithms and interactive tools for analyzing the complete dataset of projected angular separations in order to find systematic trends. Currently, most possible sources for systematic errors are being indentified by the PRIMA consortium. ${ }^{5}$ This study will lead to a series of parameters on for which trends may have to be fitted, as well as how large the systematic errors due to these parameters may be. The following steps show a simple example of how such an analysis could go:

1. Select a number of calibration measurements of known visual binaries from a sky distribution which limits the number of possible dependencies as much as possible. E.g. when trends as function of altitude are to be found then the preferable two or more binary stars with similar colors and different declination should be selected. In this case different altitudes with the same azimuth can be compared to make sure that altitude trends are distinguished from azimuth trends.

2. Compute the expected projected separations with the orbital elements of the binary stars and the baseline at the times of the observations. The orbital elements are received from one of the astrophysical tables and updated each time a calibrator is observed.

3. Plot the difference between the observed and expected projected angular separation to visually identify trends and try to fit the trend with a fitting routine.

4. Repeat from step 1 for many other selections which emphasize on other parameters. 


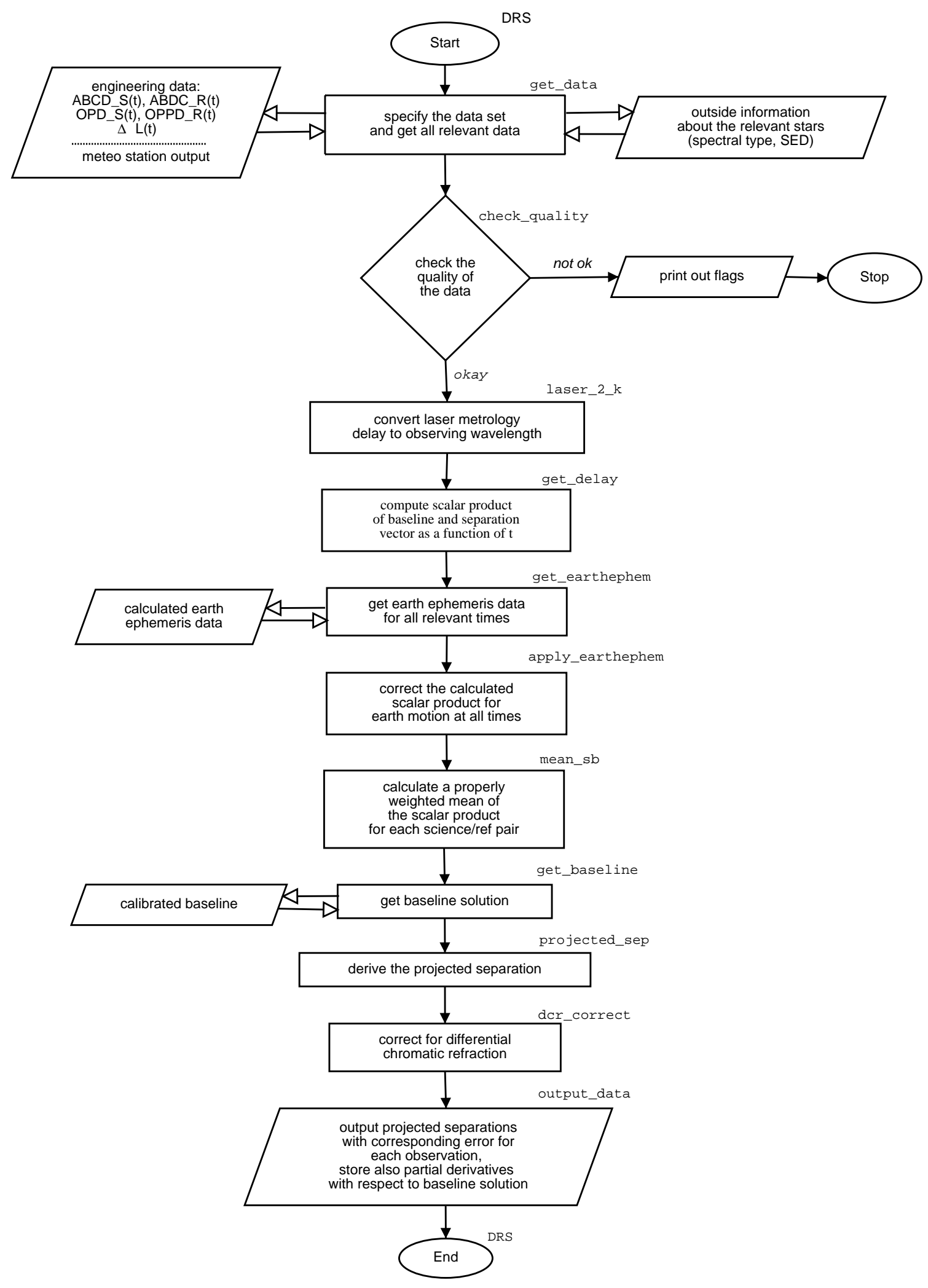

Figure 2. Preliminary diagram of the reduction recipe (from private communication with Sabine Frink). 
5. Select a number of parameters for which clear trends are visible.

6. Do a multidimensional fit on these parameters with their expected boundary conditions.

7. Write the coefficients into the database for later use by the Calibration Engine.

In practice this analysis will probably become much more complicated. Especially for the selection of proper datasets and disentangling the trends advanced optimization and search techniques may have to be utilized. Furthermore the found trends will be fed back into the reduction to provide a better calibration. After this calibration the trend analysis may have to be repeated to refine it.

APIs will be provided for the plug-ins (see Sect. 3.7) and interface to the Sequencer (see Sect. 3.5).

\subsection{Analysis Chain Manager}

The Analysis Chain Manager takes care of filling the analysis step records, which contain the associations with other steps, input parameters and results. Altogether these step records will form the analysis chain which has to be followed for a particular observation. It classifies the raw data from the FITS headers and creates for each type of observation appropriate records in the database. The classification routines will probably be obtained from the ESO Common Pipeline Library (CPL). ${ }^{4}$

\subsection{Sequencer}

The Sequencer is responsible for executing the analysis steps and updating the database with their results. Each algorithm interfaces with the Sequencer through an API which specifies the following:

1. Input parameters for optimization of the algorithm.

2. Reference files which the algorithm may require.

3. Results from previous steps which provide input to this step.

4. Where to put the results of the algorithm (files, database points).

5. Error conditions.

The algorithms will be executed by the Sequencer in the following way:

1. Check the top level step of the specified analysis. The name of the top level step is specified in the recipe configuration file.

2. Traverse from this step recursively through all dependencies. A method execute() in the PipelineCommand class creates for each dependency a new PipelineCommand object for which it invokes its execute() methods again.

3. The execute() calls the isReduced() method which checks whether the particular step already has been done, or is locked by another process.

4. In case the step is locked a wait loop will be entered until the lock has been released, which means that the other process has finished that particular reduction step.

5. A reduction step will be (re)calculated when:

(a) A file or reduction date is missing

(b) One of the dependencies is of a later date

(c) The step has been invalidated by the ACM because parameters have been changed

6. The reduction is finished when the lowest level step has been executed.

This scheme provides for an efficient way of computing the intermediate data, where steps are only recomputed when something has changed at a lower level in the analysis tree. 


\subsection{Calibration Engine}

The calibration engine computes from the latest contents of the database a set of calibration files, which has to be used in the ESO pipelines. With these calibration files any dataset should be calibrated properly to the best knowledge available at the time, and within the parameter space of the observed calibrators. The calibration engine will use the same APIs as all the other reduction/analysis steps in the chain.

\subsection{Server and user interfaces}

The server provides the only user access to the system. It runs on the Apache Tomcat web application server, ${ }^{6}$ which implements the Servlet 2.3 specification. ${ }^{7}$ The server runs several managers which manage the input data, intermediate data, running reductions and exporting the calibration data. It is accessed through a single servlet, which handles the user authentication, stores the user data and determines which application page should be displayed. All application pages are derived from a single abstract class which provides a uniform look and feel. The manager methods are called upon user actions in the application framework. Fig. 3 shows a class structure diagram of the server and underlying software layer. The following classes are defined in this diagram:

MainServlet Handles the user authentication and determines which application to show, depending on the state of the current session of the user.

AbstractApplication Framework class for a uniform look and feel of the applications in the UI.

DataSelector Deals with the selection of the data to analyse in the current session.

ObsBlockEditor Examine the observation blocks and unselect bad data.

CatalogEditor Edits catalogs of science and calibration targets.

AnalysisTools Provides access to the complete reduction/analysis chain. Shows the parameters and plug-ins of the steps and starts/monitors/aborts the analysis of certain or all OBs.

VisualizationPlugin Base class for all visualization plug-ins.

CalibrationTool User interface to the calibration engine.

ServerContainer Collection of business logic classes to be used by the UI. An instance of this container is stored in the session of the user. The container contains the main classes of the different modules of the system.

\section{IMPLEMENTATION ISSUES}

The DAF will become quite a complex system, which has to be maintained over a period of up to 10 years. This means that the code has to be well documented and conforming to the design at all times during development. Hardcoding values should be avoided and properly designed configuration files should be used where applicable. ESO library tools will be used whenever they meet the functionality for a certain module and can be easily integrated into the DAF. They should not pose limitations on the design and/or language/tool choices for the other parts of the DAF. Therefore, cross language integrations are foreseen. The Common Pipeline Library $(\mathrm{CPL})^{4}$ will most likely be integrated to provide a common codebase with the ESO pipeline, which is also part of the DRL. Furthermore, using non-ESO software packages will be investigated when no appropriate ESO library is available for a certain functionality. For example parts of the Sequencer, ACM and Database modules may be derived from the GANDALF package, ${ }^{8}$ which has been developed in Leiden for interactive pipeline reductions of Integral Field Units.

The operation maintenance will be minimized by choosing well supported and widely used software packages for the external tools. The most important external packages will be the database and the webserver, for which currently MySQL and Apache Tomcat are foreseen. These packages are nowadays included in allmost any Linux distribution and accompanied with configuration tools. An extra installation and configuration tool has to be provided for the DAF itself. A single supported Linux distribution (or other UNIX platform) has to be choosen to make sure that the configuration tool is compatible with the platform. 


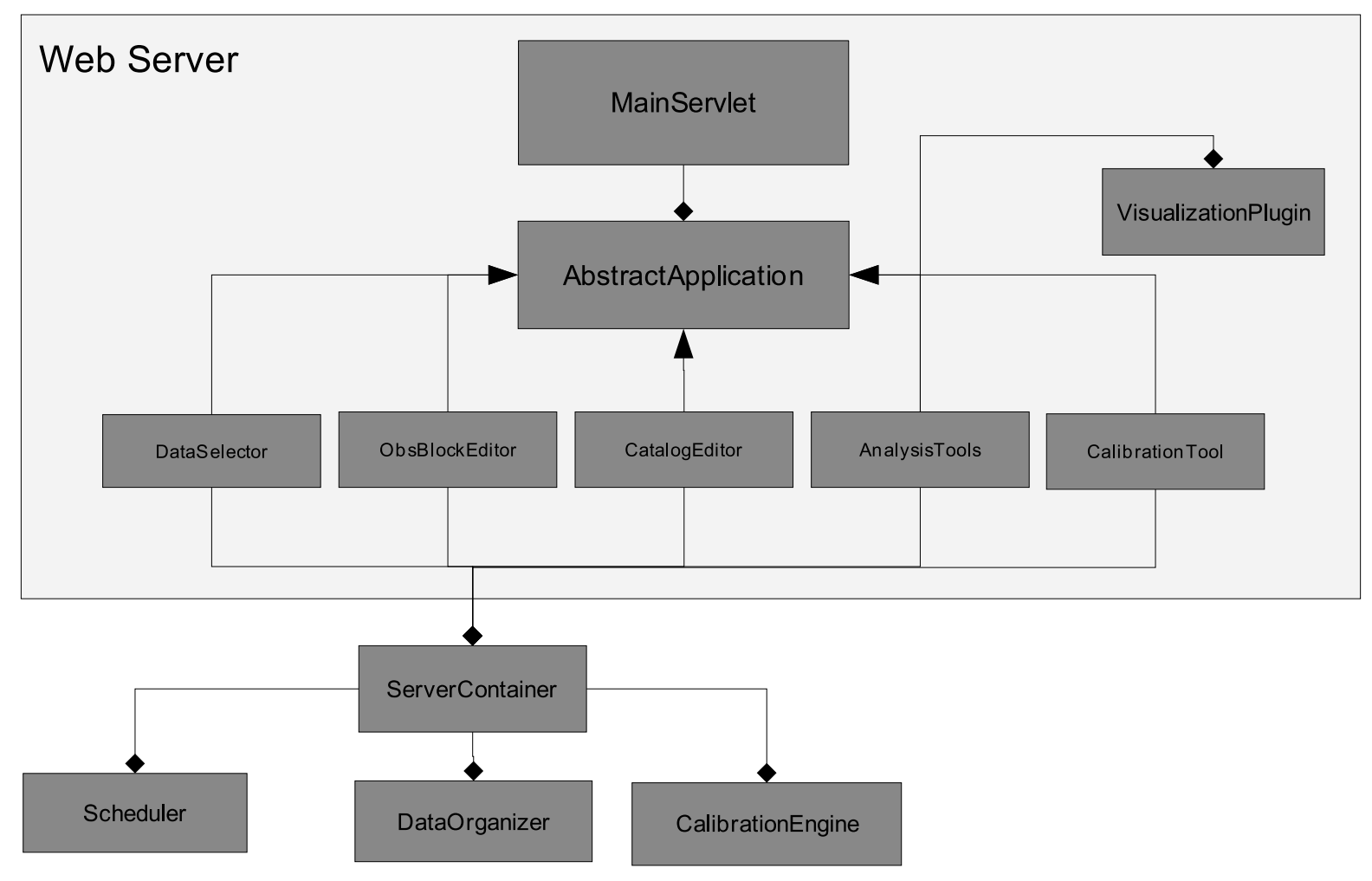

Figure 3. Class structure diagram for Server, ACM and Sequencer

\section{ACKNOWLEDGMENTS}

J.A. de Jong and E.J. Bakker are supported by NEVEC, a project of NOVA, the Research School of Astronomy in the Netherlands.

\section{REFERENCES}

1. B. Lane and M. Muterspaugh, "Differential astrometry of sub-arcsecond scale binaries at the palomar testbed interferometer," 601, 2004.

2. F. Delplancke, F. Derie, F. Paresce, A. Glindemann, F. Lévy, S. Lévêque, and S. Ménardi, "PRIMA for the VLTI - Science," Astrophysics and Space Science 286, pp. 99-104, 2003.

3. S. Frink, S. Hekker, R. Launhardt, J. Setiawan, D. Segransan, A. Quirrenbach, T. Henning, and D. Queloz, "Preparing the PRIMA Astrometric Planet Search: Selecting Suitable Target and Reference Stars ," Proceedings of the SPIE conference on New Frontiers in Stellar Interferometry 5491(130), 2004.

4. K. Banse, P. Ballester, C. Izzo, et al., "The common pipeline library: standardizing pipeline processing," Proceedings of the SPIE on Optimizing Scientific Return for Astronomy through Information Technologies 5493(34), 2004.

5. R. L. Poole, F. Delplancke, and R. Tubbs, "Phase referenced imaging and micro-arcsecond astrometry: its errors and the expected quality of their suppression," Proceedings of the SPIE conference on New Frontiers in Stellar Interferometry 5491(64), 2004.

6. "Jakarta tomcat project," http://jakarta.apache.org/tomcat/index.html, 2003.

7. "Servlet specification 2.3," http://java.sun.com/products/servlet/2.3/javadoc/index.html, 2003.

8. J. A. de Jong, R. Bacon, E. Emsellem, J. Falcón-Barosso, R. McDermid, and R. F. Peletier, "GANDALF a generic system for pipeline data reduction with interactive analysis," in preparation, 2004. 